\title{
Adaptive Transmission Policy for Energy Harvesting Relaying systems
}

\author{
Abdelhamid Salem, and Leila Musavian \\ School of Computer Science and Electronic Engineering, University of Essex, Colchester, UK. \\ Emails: \{abdelhamid.salem, Leila.musavian\}@essex.ac.uk.
}

\begin{abstract}
In this paper, we consider an energy harvesting (EH)-based relaying system where an EH-source node equipped with a rechargeable battery to store the energy harvested from the environment, communicates with a destination with the help of a relay node. The relay and destination both have an unlimited power supply, while the source relies solely on the harvested energy. A delay-limited transmission mode is assumed in this paper, in which if the source data cannot be transmitted within a delay deadline, it will be lost. Based on this model, an efficient adaptive source transmission policy is proposed. Markov chain analysis is considered to model the levels of the stored energy at the source node and the system performance is evaluated in terms of the transmission and success probabilities. The results reveal that the benefit of the proposed transmission strategy in delaylimited applications is highly dependent on the proper choice of the system design parameters and the harvested energy per packet.
\end{abstract}

Index Terms-Energy harvesting, cooperative communications, Markov chain, amplify-and-forward, relay.

\section{INTRODUCTION}

Energy harvesting (EH) communication systems have attracted significant research attention in recent years. In these systems, all the nodes equipped with EH devices are able to harvest energy from the surrounding environment, for instance by using solar panels [1]. These advantages are particularly attractive in applications where battery limited nodes are not easily accessible, such as wireless sensor nodes operating in hazardous areas. In recent years, much work has been done investigating EH-based wireless communication systems. The authors in [1] studied an approach to find optimal transmission policies for solar-powered sensor nodes by adapting the transmission parameters to the changes in channel fading and battery recharge. In [2] a multi-tier up-link EH cellular network was analyzed and the performance of the network evaluated by considering stochastic geometry. In [3] the authors considered a multiple access channel, where multiple users communicate with an access point (AP) using EH powered batteries, in this model the $\mathrm{EH}$ processes were assumed to be known to the users before transmissions. An optimal transmission policy for EH point-to-point wireless communications where the source is a solar-powered node was considered in [4]. Reliable communication over an additive white Gaussian noise channel in sensor networks using EH sensor nodes was studied in [5]. In [6], delay optimal power control for an EH wireless network with finite energy storage was considered, where the system is powered only by a renewable energy source with busty data arrival. The article in [7] reviews existing research on resource allocation in EH wireless systems.

The efficiency of EH in cooperative communication systems has been explored by many researchers. For instance, the work in [8] investigated the performance analysis of cooperative networks aided by EH relay nodes in terms of outage over Rayleigh fading channels. In [9] the authors considered optimal power allocation for both conventional and buffer-aided energy harvesting relay networks, where an EH source node communicates with destination nodes through an EH decode and forward (DF) relay node over multiple fading channels. Transmission policies for EH wireless sensor networks have been addressed in [10], where the nodes may use either direct transmission or cooperative relaying. In [11], a cooperative system in which EH nodes can serve as relays when they had sufficient energy for transmission was studied. Furthermore, in [12] the authors considered the use of energy harvesters in wireless cooperative communication, in which the source and relay nodes transmit the data using the power harvested from EH sources under the assumption of a deterministic EH model, i.e., the amount of energy and energy-arrival time are both known. In [13] an optimal relay transmission policy in two-way relay network was proposed, in this model the relay is solar powered and equipped with a finite size battery to store the energy.

In this paper, we consider an efficient transmission strategy for an EH relaying system where the source is an EH node equipped with a finite-sized rechargeable battery, while the relay and the destination both have fixed power supplies. A delay-limited transmission mode is assumed in this model, in which the source is obliged to send delay-constrained data periodically. If the source signals cannot be sent within a specific delay deadline, the signals will be assumed lost. In this model the source can know the statistics of the harvested energy [12]; in practical applications, for instance, the solar EH state can be estimated and updated using the real data of solar irradiation at the source [1]. Based on this knowledge, the relay can be located in a position where one unit of harvested energy is sufficient to transmit data from the source to relay node. Therefore, the transmission strategy in this system is achieved as follows, if the harvested energy is sufficient for source to destination transmission, then the source will transmit the data directly to the destination, otherwise the source will transmit the data to the destination through the relay node with a lower data rate. Such scenarios occur in general wireless sensor networks whose function is to monitor 


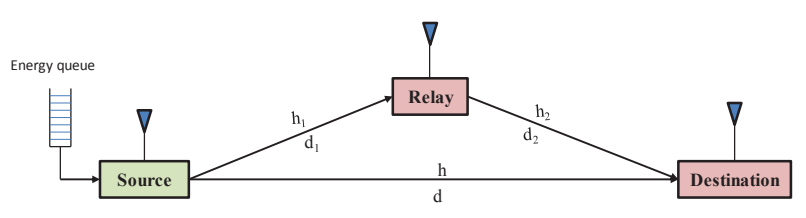

Figure 1: Relaying system with EH source node.

and report important events in real time. The performance of the proposed model is evaluated in terms of the transmission and success probabilities using a Markov chain. Therefore, in this paper we first derive analytical expressions for the transmission and success probabilities of the proposed system. We then examine the impact of various system parameters on the system performance.

The rest of this paper is organized as follows. Section II describes the system model under consideration. Section III derives analytical expressions for the transmission probability. Section IV derives analytical expressions for the success probability. Numerical examples and simulation results are presented and discussed in section V. Finally, Section VI draws the main conclusions.

\section{System Model}

We consider an AF relaying system consisting of a single antenna source node sending information signal to a single antenna destination node in the presence of a single antenna relay node. On one hand, the relay and destination both have unlimited power supply. On the other hand, the source is EH node which relies solely on energy harvested from the environment, for instance solar panels [1]. Therefore, the amount of harvested energy at the source is not fixed and varies over time based on the variations of the natural sources. The channel coefficients between the nodes are shown in Fig. 1, where $h$ is the source-to-destination channel, $h_{1}$ is the sourceto-relay channel and $h_{2}$ is the relay-to-destination channel; all the channels are modeled as quasi-static block fading channels, i.e., the channels are assumed to be constant over block time and vary independently and identically from one block to another, following a Rayleigh distribution magnitude. The distances from the source to destination, source to relay, relay to destination nodes are represented by $d, d_{1}$ and $d_{2}$, respectively.

The system is considered to be time slotted, as shown in Fig. 2 , the length of each slot is equal to $T_{n}$ seconds and the source battery is recharged once at the beginning of each time slot $n(n=1,2, \cdot \cdot)$. According to discrete-time energy arrivals, in time slot $n$ the source receives $m^{(n)}$ energy packets, with one energy packet contains $E_{k}$ (Joule), where $m^{(n)} \in\{0,1, \cdots\}$ is a random number. Without loss of generality, it is assumed that the number of energy packets arriving at the source follows a Poisson distribution with the parameter $\lambda_{e}$ [3]. Taking into account the efficiency of the storage and the impact of energy leakage, the total amount of the stored energy at the source in slot $n$ can be given by, $E^{(n)}=m^{(n)} E_{k} \mu_{k}$ (Joule), where $\mu_{k}$ presents the practical factor for battery usage. Therefore, the energy storage process can be modeled as an energy queue.
The system model under consideration is for applications in which the source can estimate the $\mathrm{EH}$ profile accurately. In practical applications, for instance, the solar EH state can be estimated and updated using the real data of solar irradiation at the source, and the current channel and battery states can be easily obtained [1]. Based on this information the system is designed to locate the relay such that the source can communicate with the relay by consuming only one energy packet $E_{k}$. Thus, communication in the proposed system is achieved as follows: if the harvested energy at the transmission time is enough for direct transmission, the source transmits the data directly to the destination. On the other hand, if there is no enough energy for direct transmission the source transmits the data to the destination through the relay node with a lower rate and consuming only one unit of energy.

\section{TRANSMISSION PROBABILITY}

Based on the system model described above, we can define the required amount of transmission power for the source to relay $\gamma_{r}$ and source to destination $\gamma_{d}$, respectively, as [2]

$$
\gamma_{r}=\xi_{r} d_{1}^{\varpi} \text { and } \gamma_{d}=\xi_{d} d^{\varpi},
$$

where $\varpi$ is the path loss exponent and $\xi_{r}, \xi_{r}$ are constants. Consequently, the transmission probabilities of the source to relay $\left(\eta_{r}\right)$ and to the destination $\left(\eta_{d}\right)$ are given by

$$
\eta_{r}=\operatorname{Pr}\left(P_{s}(T)>\gamma_{r}\right), \eta_{d}=\operatorname{Pr}\left(P_{s}(T)>\gamma_{d}\right),
$$

where $P_{s}(T)$ is the amount of the stored power during time period $T$, and it is given by $P_{s}(T)=\sum_{t=0}^{T} P_{H}(t)$, while $P_{H}(t)$ is the harvested power at time $t$. This definition of the transmission probability can be explained as the harvested power at the source should be larger than $\gamma_{r}$ for sourcerelay transmission, and larger than $\gamma_{d}$ for source-destination transmission. As we know from the previous section the number of energy packets arriving at the source follows a Poisson distribution, therefore, $m$ energy packets can be harvested by the source with probability, $f_{s}(m)=\frac{e^{-\lambda_{e}} \lambda_{e}^{m}}{m !}$, where $m=0,1,2, \ldots$.

In order to simplify the model, the level (or the state) of the source battery is modeled simply as a one-dimensional random walk which is then modeled by a finite-state Markov chain. The battery is divided into a finite number of levels and the state space can be considered as a finite set. This stochastic process demonstrates the Markov property that, at a given time slot, the state of the battery depends only on the level in the previous time slot. Based on this model we can define $L$ and $w$ as the total number of the battery levels and the step size, respectively, and then the battery $\operatorname{size}(B)$ can be given by, $B=L w$. In addition, we can also define $p_{i}$ as the probability that the amount of harvested power at the source in a certain time slot is $i w$ Watt, i.e., $p_{i}=\operatorname{Pr}\left[P_{H}=i w\right]$ for $i=$ $\{0,1,2, \ldots, L-1\}$ and $p_{L}=\operatorname{Pr}\left[P_{H} \geq B=L w\right]$. On the other hand, we assume the data arrival in each slot has a Bernoulli distribution, with probability of occurrence $q$. According to these energy levels, the communication between the source 


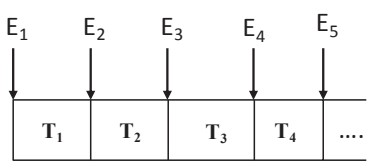

Figure 2: Energy Arrivals.

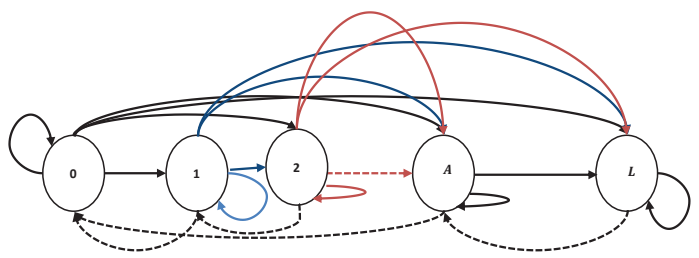

Figure 3: Markov chain model of the battery states for $L+1$ discrete levels.

and the relay is possible by consuming $1 w$ (one step, $L=1$ ) and the communication between the source and the destination is possible by consuming $A w$, where $1<A<L$. Therefore, if the battery state is less than $A w$ the source will decide to transmit the data to the relay node and consume $1 w$ with a lower rate, and if the battery state is more than $A w$ the source will decide to transmit the data to the destination and consume $A w$ with a higher rate.

Using the definitions of $p_{i}$ and $q$ with the transmission policy discussed, we can plot the state diagram of the finite state Markov Chain as in Fig. 3 and the transition probability matrix $\mathrm{P}$ as in (3), where $\mathrm{P} i, j$ is the transition probability for one step, i.e, from state $i$ to state $j$. Note that, in our model, the battery has a total of $L+1$ different energy levels or states, where the $l^{\text {th }}$ state indicates a battery level is $l w$ Watt. In Fig. 3, the transition from a higher level to a lower level shows that the source is in transmission mode while the transition from lower to a higher level or remaining at the same level indicates that the source is in energy harvesting mode and there is no data transmission.

Since the Markov Chain for this model is homogeneous and irreducible, steady-state probability vector can be found by solving a set of linear equations. First, we define $\pi=$ $\left[\pi_{0}, \pi_{1}, \ldots \ldots, \pi_{L}\right]$ as the steady state vector, where $\pi_{l}$ is the probability that the Markov Chain is in state $l$ and $\sum_{l=0}^{L} \pi_{l}=1$. Then we can write the set of equations as

$$
\pi=\pi \mathrm{P} \text { and } \mathbf{1}=\pi \mathbf{1}
$$

where $\mathbf{1}=[1,1, \ldots \ldots .1]^{T},[.]^{T}$ is the transpose operation and $\sum_{j=0}^{L} P_{i j}=1$. In order to find $\pi_{l}$, we should solve the linear equations in (4). Consequently, the probability that the amount of energy stored in the battery is sufficient to transmit the data to the relay and the destination are given, respectively, by

$$
\eta_{r}=\sum_{l=1}^{A-1} \pi_{l} \text { and } \eta_{d}=\sum_{l=A}^{L} \pi_{l}
$$

\section{Success Probability}

The success probability $(s p)$ is the probability that, the amount of the energy stored in the source's battery is sufficient for data transmission and the level of the signal to noise ratios (SNR) at the destination is larger than a predefined threshold value. The mathematical expression of the success probability is given by

$$
s p=\eta_{r} C_{1}+\eta_{d} C_{2},
$$

where $C_{1}=\operatorname{Pr}\left(\gamma_{r, d}>\alpha_{r, d}\right), C_{2}=\operatorname{Pr}\left(\gamma_{d}>\alpha_{d}\right), \gamma_{r, d}$ is the SNR at the destination for source-relay-destination link, $\alpha_{r, d}$ is threshold value of the SNR for source-relay-destination link, $\gamma_{d}$ is the SNR at the destination for source-destination link, $\alpha_{d}$ is threshold value of the SNR for source-destination link and $\eta_{r}, \eta_{d}$ are given by (5), respectively. Hence, we can write (6) as

$$
s p=\sum_{l=1}^{A-1} \pi_{l} C_{1}+\sum_{l=A}^{L} \pi_{l} C_{2} .
$$

To derive $C_{1}$ we can write, $C_{1}=1-\bar{C}_{1}$, where $\bar{C}_{1}$ is the outage probability and given by

$$
\bar{C}_{1}=\operatorname{Pr}\left(\gamma_{r, d}<\alpha_{r, d}\right) \text {. }
$$

The SNR at the destination for source-relay-destination $\gamma_{r, d}$ is given by

$$
\gamma_{r, d}=\frac{P_{s, l} G_{r}^{2}\left|h_{1}\right|^{2}\left|h_{2}\right|^{2}}{G_{r}^{2} d_{1}^{\varpi}\left|h_{2}\right|^{2} \sigma_{r}^{2}+d_{1}^{\varpi} d_{2}^{\varpi} \sigma_{d}^{2}},
$$

where $P_{s, l}=1 w$ and $\sigma_{r}^{2}, \sigma_{d}^{2}$ are the noise variances at the relay and the destination nodes, respectively, and $G_{r}$ is a constant relay gain, $G_{r}=\sqrt{\frac{P_{r}}{P_{s, l} d_{1}^{-\varpi}+\sigma_{r}^{2}}}$. Substituting (9) into (8) we get

$$
\bar{C}_{1}=\operatorname{Pr}\left(\left|h_{1}\right|^{2}<\frac{\alpha_{r, d} d_{1}^{\varpi} \sigma_{r}^{2}}{P_{s, l}}+\frac{d_{1}^{\varpi} d_{2}^{\varpi} \alpha_{r, d} \sigma_{d}^{2}}{P_{s, l} G_{r}^{2}\left|h_{2}\right|^{2}}\right) .
$$

Therefore,

$$
\bar{C}_{1}=\int_{0}^{\infty} F_{\left|h_{1}\right|^{2}}\left(\frac{\alpha_{r, d} d_{1}^{\varpi} \sigma_{r}^{2}}{P_{s, l}}+\frac{d_{1}^{\varpi} d_{2}^{\varpi} \alpha_{r, d} \sigma_{d}^{2}}{P_{s, l} G_{r}^{2} x}\right) f_{\left|h_{2}\right|^{2}}(x) d x
$$

where $F_{X}(x)$ is the commutative distribution function (CDF) of $X$ and $f_{Y}(y)$ is the probability density function (PDF) of $Y$. Since $\left|h_{1}\right|^{2}$ and $\left|h_{2}\right|^{2}$ have exponential distributions, the CDF and PDF are given by, respectively, $F_{\left|h_{1}\right|^{2}}\left(x_{1}\right)=$ $\left(1-e^{-x_{1}}\right)$ and $f_{\left|h_{2}\right|^{2}}\left(x_{2}\right)=e^{-x_{2}}$. Therefore, (11) can be written as

$$
\bar{C}_{1}=\int_{0}^{\infty}\left(1-e^{-\left(\frac{\alpha_{r, d} d_{1}^{\varpi} \sigma_{r}^{2}}{P_{s, l}}+\frac{d_{1}^{\varpi} d_{2}^{\varpi} \alpha_{r, d} \sigma_{d}^{2}}{P_{s, l} G_{r}^{2} h}\right)}\right) e^{-x} d x
$$

$\bar{C}_{1}=1-2 \sqrt{\frac{d_{1}^{\varpi} d_{2}^{\varpi} \alpha_{r, d} \sigma_{d}^{2}}{P_{s, l} G_{r}^{2}}} e^{-\frac{\alpha_{r, d} d_{1}^{\varpi} \sigma_{r}^{2}}{P_{s, l}}} \mathrm{~J}\left[1,2 \sqrt{\frac{d_{1}^{\varpi} d_{2}^{\varpi} \alpha_{r, d} \sigma_{d}^{2}}{P_{s, l} G_{r}^{2}}}\right]$, 
$\mathbf{P}=\left[P_{i . j}\right]$

$$
\mathrm{P}=\left[\begin{array}{ccccccccc}
p_{0} & p_{1} & p_{2} & . . & p_{A-1} & p_{A} & p_{A+1} & . . & p_{L} \\
q & p_{0}(1-q) & p_{1}(1-q) & . & p_{A-2}(1-q) & p_{A-1}(1-q) & p_{A}(1-q) & . . & \left(p_{L}+p_{L-1}\right)(1-q) \\
0 & q & p_{0}(1-q) & . . & p_{A-3}(1-q) & p_{A-2}(1-q) & p_{A-1}(1-q) & . & \left(p_{L}+p_{L-1}+p_{L-2}\right)(1-q) \\
: & : & : & : & : & : & : & : & : \\
: & : & : & : & : & : & : & : & : \\
0 & 0 & 0 & . . & p_{0}(1-q) & p_{1}(1-q) & p_{2}(1-q) & . . & \left(p_{A+1}+\ldots+p_{L}\right)(1-q) \\
q & 0 & 0 & . & 0 & p_{0}(1-q) & p_{1}(1-q) & . & \left(p_{A}+\ldots+p_{L}\right)(1-q) \\
: & : & : & : & : & : & : & : & : \\
: & : & : & : & : & : & : & : & : \\
: & : & : & : & : & : & : & : & (1-q) \\
0 & 0 & 0 & . . & 0 & q & 0 & . . & :
\end{array}\right] .
$$

where $\mathbf{J}[$.$] is the Bessel function of the second kind. To derive$ $C_{2}$, we can write, $C_{2}=1-\bar{C}_{2}$, where $\bar{C}_{2}$ is the outage probability and given by,

$$
\bar{C}_{2}=\operatorname{Pr}\left(\gamma_{d}<\alpha_{d}\right) .
$$

The SNR at the destination for source-destination $\operatorname{link} \gamma_{d}$ is given by

$$
\gamma_{d}=\frac{P_{s, l}|h|^{2}}{d^{\varpi} \sigma_{d}^{2}},
$$

where $P_{s, l}=A w$. Substitute (15) into (14), we get,

$$
\bar{C}_{2}=\operatorname{Pr}\left(|h|^{2}<\frac{\alpha_{d} d^{\varpi} \sigma_{d}^{2}}{P_{s, l}}\right) .
$$

Since $|h|^{2}$ has exponential distribution, this last equation can be written as

$$
\bar{C}_{2}=1-e^{-\left(\frac{\alpha_{d} d^{\varpi} \sigma_{d}^{2}}{P_{s, l}}\right)} .
$$

Finally, the probability of success can be written as in (18), shown at the top of the next page.

\section{NUMERICAL RESULTS}

In this section, we present some numerical results for the analytical expressions derived above. The impact of different system parameters on the performance metrics, transmission probabilities and the success probability will be investigated. Without loss of generality, we assume that, $A=\frac{L}{2}$, the source energy packets contain an equal amount of power, $E_{k}=0.05 \mathrm{~W}, d_{1}=1 \mathrm{~m}, d_{2}=4 \mathrm{~m}, d=5 \mathrm{~m}$, the pathloss exponent is $\varpi=2.7$ and $\alpha_{r, d}=\alpha_{d}=\alpha$. The source battery capacity is assumed to be $27 \mathrm{dBm}$ and the number of levels for the battery states is $10, \mu=1$ and the relay power $P_{r}=50 \mathrm{dbm}$.

In Fig. 4, the transmission probability is plotted against the amount of harvested energy per packet $E_{k}$, when $d=10 \mathrm{~m}$, $d_{1}=5 \mathrm{~m}$ and $d_{2}=5 \mathrm{~m}$. As we can see from the figure, when $E_{k}$ is small the transmission through the relay has higher probability than direct transmission, i.e., $\eta_{r}>\eta_{d}$; increasing $E_{k}$ increases the probability of direct transmission and decreases the probability of transmission via the relay, i.e., when $E_{k}$ is larger than $0.01, \eta_{d}$ will be higher than $\eta_{r}$, $\eta_{d}>\eta_{r}$.

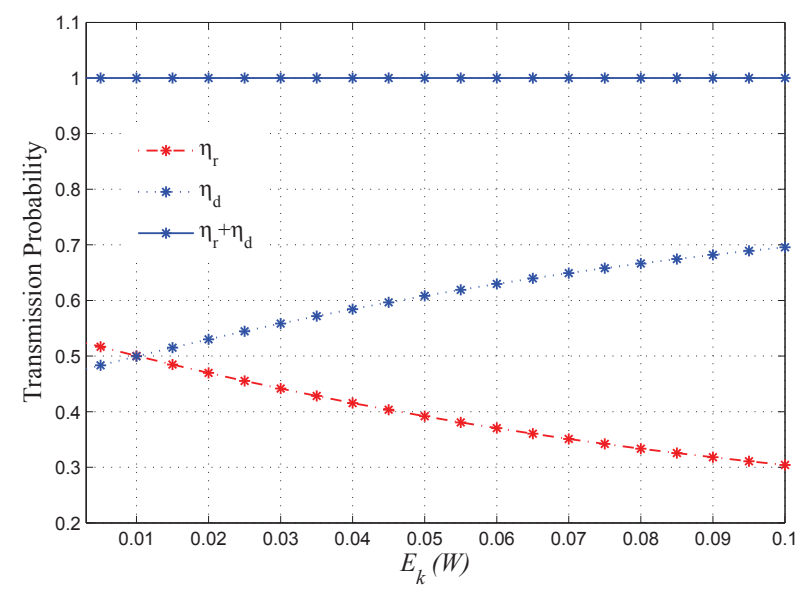

Figure 4: Transmission probability versus $E_{k}$.

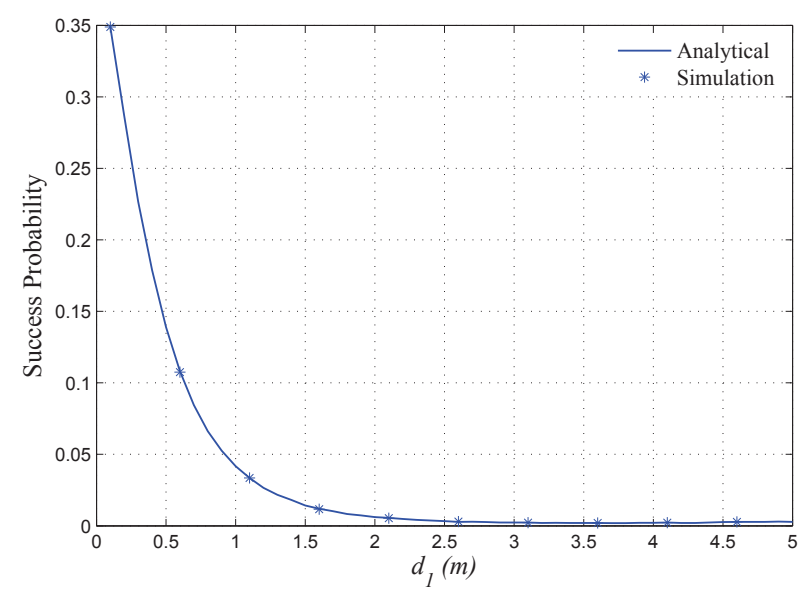

Figure 5: Success probability versus the source-relay distance.

In Fig. 5, we plot the success probability versus the distance between the source and the relay $d_{1}$ when $\alpha=1$, $P_{r}=31.76 \mathrm{dbm}, d=5 \mathrm{~m}$ and $d_{2}=d-d_{1} \mathrm{~m}$. From this figure, it is clear that the best location of the relay is when it is close to the source node, but if the relay is located more than 2 meters away from the source the success probability will be zero. Therefore, in the delay-limited transmission mode the relay should be located no further than $2 \mathrm{~m}$ away from the source.

In Fig. 6, we plot the success probability as a function of the 


$$
s p=\left(\sum_{l=1}^{A-1} \pi_{l}\right)\left(2 \sqrt{\frac{d_{1}^{\varpi} d_{2}^{\varpi} \alpha_{r, d} \sigma_{d}^{2}}{P_{s, l} G_{r}^{2}}} e^{-\frac{\alpha_{r, d} d_{1}^{\varpi} \sigma_{r}^{2}}{P_{s, l}}} \mathrm{~J}\left[1,2 \sqrt{\frac{d_{1}^{\varpi} d_{2}^{\varpi} \alpha_{r, d} \sigma_{d}^{2}}{P_{s, l} G_{r}^{2}}}\right]\right)+\left(\sum_{l=A}^{L} \pi_{l}\right) e^{-\left(\frac{\alpha_{d} d^{\varpi} \sigma_{d}^{2}}{P_{s, l}}\right)} .
$$

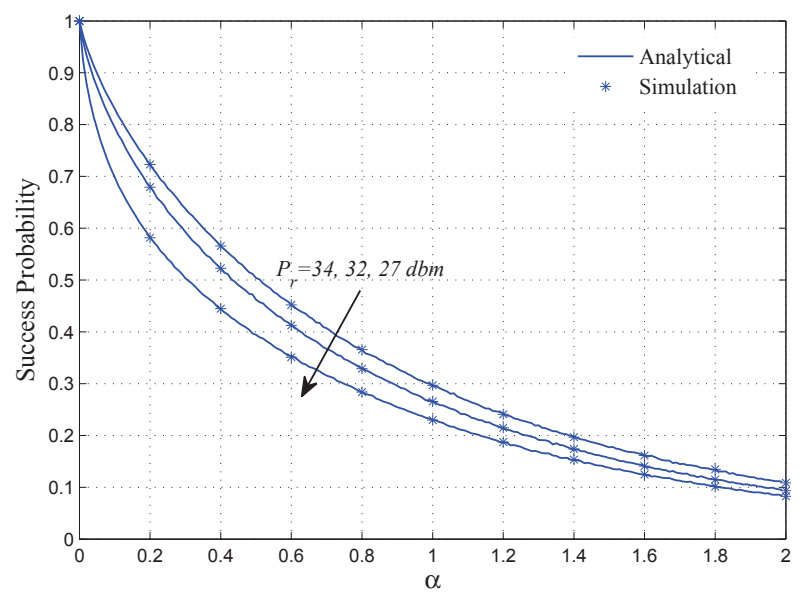

(a) Success probability versus $\alpha$ for different values of $P_{r}$.

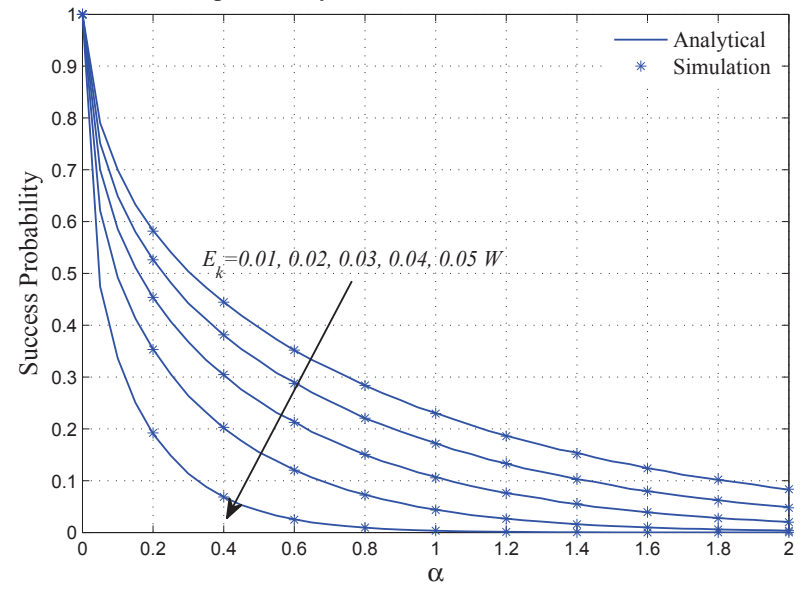

(b) Success probability versus $\alpha$ for different values of $E_{k}$.

Figure 6: Success probability versus the threshold value $\alpha$ for different values of $P_{r}$ and $E_{k}$.

threshold value $\alpha$ for different values of the relay power, $P_{r}$ and $E_{k}$. As we can see from the two sub-figures, the success probability is high when the threshold value, $\alpha$, is small and deteriorates with increasing $\alpha$.

In Fig. 6a, it can also be seen that the gap between the $P_{r}=32 \mathrm{dbm}$ and $P_{r}=34 \mathrm{dbm}$ is tighter than that between $P_{r}=27 \mathrm{dbm}$ and $P_{r}=32 \mathrm{dbm}$. Therefore, increasing the relay power to higher values might not increase the system performance much.

In order to show the impact of the amount of energy harvested per packet $E_{k}$ on the system performance, in Fig. $6 \mathrm{~b}$ we plot the success probability versus the threshold value $\alpha$ for different values of $E_{k}$. As we can see form the figure that the success probability is highly dependent on the amount of harvested energy per packet, and this is because when $E_{k}$ is high, the direct transmission probability $\eta_{d}$ will be high and therefore the system performance can be greatly enhanced.

\section{CONCLUSIONS}

In this paper, we studied a transmission strategy for an EH relaying system, where the source is an $\mathrm{EH}$ node equipped with a finite-sized battery. A delay-limited transmission mode was considered, in which the source can ascertain the statistics of the EH. The performance of the proposed system was studied using a Markov chain approach. The results showed that the benefit of the proposed transmission strategy in delay-limited applications is highly dependent on the energy harvested per packet and the system design parameters.

\section{REFERENCES}

[1] M. L. Ku, Y. Chen, and K. J. R. Liu, "Data-driven stochastic models and policies for energy harvesting sensor communications," IEEE Journal on Selected Areas in Communications, vol. 33, pp. 1505-1520, Aug 2015.

[2] A. H. Sakr and E. Hossain, "Analysis of k -tier uplink cellular networks with ambient rf energy harvesting," IEEE Journal on Selected Areas in Communications, vol. 33, pp. 2226-2238, Oct 2015.

[3] J. Liu, H. Dai, and W. Chen, "On throughput maximization of time division multiple access with energy harvesting users," IEEE Transactions on Vehicular Technology, vol. 65, pp. 2457-2470, April 2016.

[4] W. Li, M. L. Ku, Y. Chen, and K. J. R. Liu, "On outage probability for stochastic energy harvesting communications in fading channels," IEEE Signal Processing Letters, vol. 22, pp. 1893-1897, Nov 2015.

[5] R. Rajesh, V. Sharma, and P. Viswanath, "Capacity of gaussian channels with energy harvesting and processing cost," IEEE Transactions on Information Theory, vol. 60, pp. 2563-2575, May 2014.

[6] F. Zhang and V. K. N. Lau, "Closed-form delay-optimal power control for energy harvesting wireless system with finite energy storage," IEEE Transactions on Signal Processing, vol. 62, pp. 5706-5715, Nov 2014.

[7] F. Zhang and V. K. N. Lau, "Delay-sensitive dynamic resource control for energy harvesting wireless systems with finite energy storage," IEEE Communications Magazine, vol. 53, pp. 106-113, August 2015.

[8] T. Li, P. Fan, and K. B. Letaief, "Outage probability of energy harvesting relay-aided cooperative networks over rayleigh fading channel," IEEE Transactions on Vehicular Technology, vol. 65, pp. 972-978, Feb 2016.

[9] I. Ahmed, A. Ikhlef, R. Schober, and R. K. Mallik, "Power allocation for conventional and buffer-aided link adaptive relaying systems with energy harvesting nodes," IEEE Transactions on Wireless Communications, vol. 13, pp. 1182-1195, March 2014.

[10] H. Li, N. Jaggi, and B. Sikdar, "Relay scheduling for cooperative communications in sensor networks with energy harvesting," IEEE Transactions on Wireless Communications, vol. 10, pp. 2918-2928, September 2011.

[11] B. Medepally and N. B. Mehta, "Voluntary energy harvesting relays and selection in cooperative wireless networks," IEEE Transactions on Wireless Communications, vol. 9, pp. 3543-3553, November 2010.

[12] C. Huang, R. Zhang, and S. Cui, "Throughput maximization for the gaussian relay channel with energy harvesting constraints," IEEE Journal on Selected Areas in Communications, vol. 31, pp. 1469-1479, August 2013.

[13] W. Li, M. L. Ku, Y. Chen, and K. J. R. Liu, "On outage probability for two-way relay networks with stochastic energy harvesting," IEEE Transactions on Communications, vol. 64, pp. 1901-1915, May 2016. 